\title{
Indocyanine green elimination measurement may detect surgical complications following major liver resection. Preliminary results.
}

\section{Ll.Alarcón Pérez, P. Bono Chaure, J. Narváez Salazar, L. Olmedilla, JM Pérez-Peña, P. Duque González. Hospital General Universitario Gregorio Marañón, Madrid}

\section{Background and Goal of Study:}

Indocyanine green elimination by pulse spectrophotometry is a non-invasive liver function monitoring system.

The aim of this study is to assess the relationship between postoperative ICG plasma disappearance rate (ICG PDR \% min) and the onset of surgical complications postoperatorias.

\section{Materials and Methods:}

This study is registered in ClinicalTrials.gov under the number NCT02813538.

22 patients scheduled for mayor liver resection were selected. ICG PDR was measured preoperatively and on the day 1 and 2 postoperatively. We considered liver ischemia, biliary leak and intra-abdominal fluid collections as postoperative surgical complications.

\section{Results and Discussion:}

6 patients have undergone surgical complications.

The median ICG PDR was $17.2 \pm 7.7$ preoperatively, $16.96 \pm$ 7.75 on postoperative day 1 and $16.24 \pm 7.91$ on postoperative day 2. Significant association was found between lower ICG PDR measurement taken on postoperative day 1 and the onset of surgical complications $(11,3 \pm 5.9$ in patients with surgical complications vs $18 \pm 7.14$ in patients without surgical complications, $\mathrm{p}=0.017$ ).

We have also found a significant correlation between ICG PDR measurement taken on postoperative day 1 and hospital stay $(\mathrm{r}=0.43, \mathrm{p}=0.044)$.
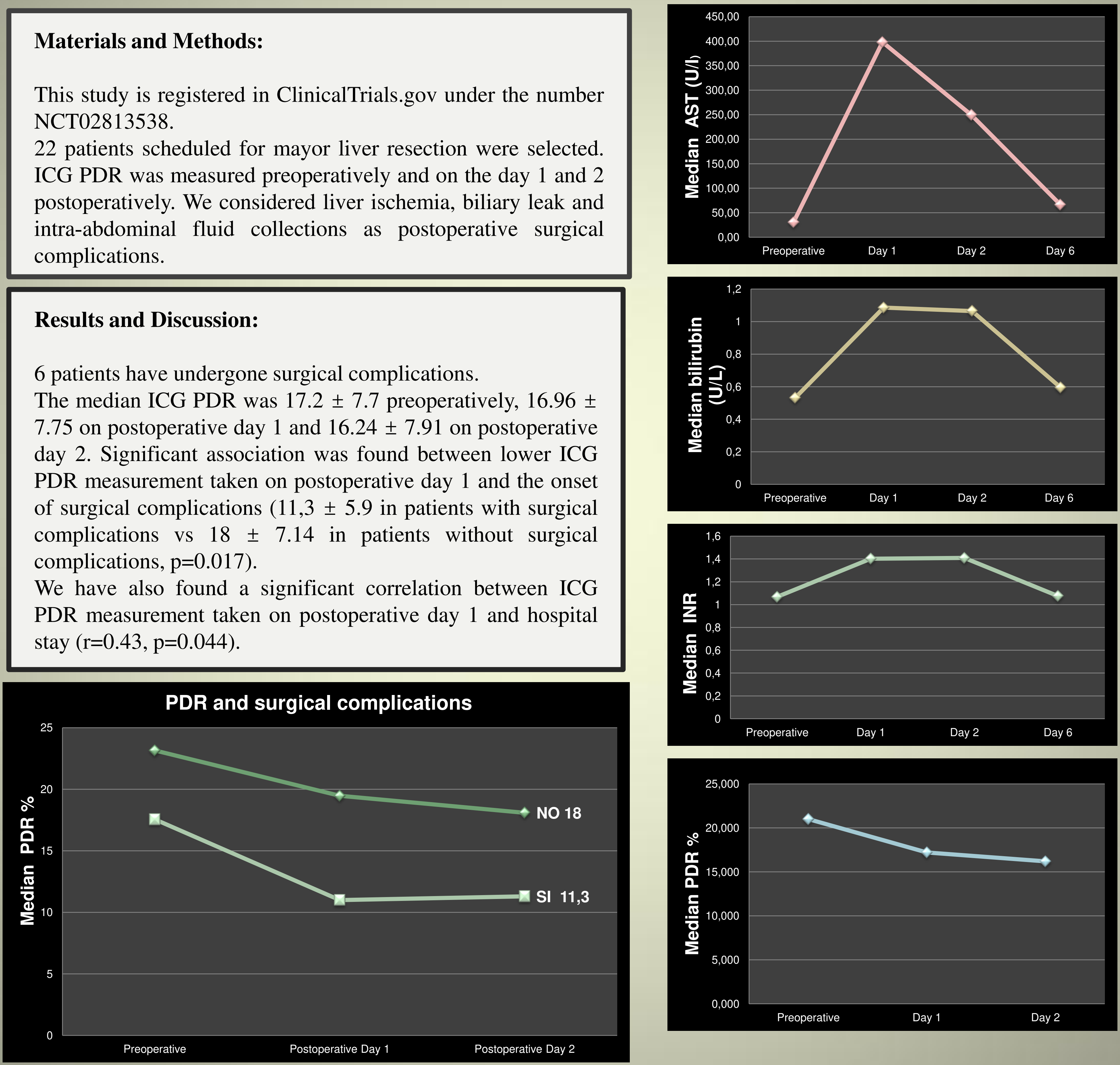

\section{Conclusions:}

ICG PDR measured by pulse spectrophotometry is a useful non-invasive liver function test in patients undergoing major liver resection. It may predict surgical complications in these patients which could improve their management and as a consequence their outcome.

References:

1.Kim HJ, Kim CY, Park EK et al. Volumetric analysis and indocyanine green retention rate at 15 min as predictors of post-hepatectomy liver failure. HPB (Oxford) 2015;17:159-67 2.Derpapas MK, Contis J, Fragulidis GP et al. Correlation of the ICG test with risk factors and postoperative outcomes following hepatic resection. JBUON 2013;18:703-7

3.de Liguori Carino N, O'Reilly DA, Dajani K et al. Perioperative use of the LiMON method of indocyanine green elimination measurement for the prediction and early detection of posthepatectomy liver failure. Eur J Surg Oncol 2009;35:957-62 